\title{
The Comparison of Blood Lipid Profile in Patients with and Without Androgenetic Alopecia
}

\author{
(D) Tuğba Akın, (1) Zekayi Kutlubay, (1) Özge Așkın
}

Istanbul University-Cerrahpasa, Cerrahpasa Faculty of Medicine, Department of Dermatology, Istanbul, Turkey

\section{ABSTRACT}

Background: Androgenetic alopecia (AGA) is the most common cause of hair loss in both sexes. In several studies investigating the relationship between AGA and dyslipidemia, conflicting results have been reported. In this study, we aimed to compare serum triglyceride (TG) and highdensity lipoprotein (HDL) cholesterol levels that one of the criteria of metabolic syndrome in male patients with and without AGA.

Materials and Methods: The study group was consisted of 40 patients who had 2 and higher AGA. The control group was consisted of 40 patients aged who had no AGA at the clinical examination or who had stage 1 AGA. Fasting serum TG and HDL cholesterol levels were compared between study and control groups.

Results: The mean serum TG values in the study group were 89.60; the mean serum TG values in the control group were 85.40. There was no statistical difference in serum TG values between the two groups $(p<0.005)$. The number of patients with serum TG value $\geq 150 \mathrm{mg} / \mathrm{dL}$ was $5(12.5 \%)$ in the study group and $3(7.5 \%)$ in the control group; however, this difference was not statistically significant too $(p<0.005)$. Mean serum HDL cholesterol levels in the study group were 52.67; the mean serum HDL cholesterol values in the control group were 52.62. There was no significant difference in serum HDL cholesterol levels between the two groups $(p<0.005)$. The number of patients with serum HDL cholesterol $<40 \mathrm{mg} / \mathrm{dL}$ was $4(10 \%)$ in the patient group and $1(2.5 \%)$ in the control group; however, this difference was not statistically significant too $(p<0.005)$.

Conclusion: There was no significant difference between serum TG and HDL cholesterol levels in male patients with or without AGA.

Keywords: Androgenetic alopecia, Dyslipidemia, Triglyceride, High-density lipoprotein cholesterol

\section{Introduction}

As the name suggests, androgenetic alopecia (AGA) is a hair loss with a specific clinical pattern, characterized by follicular miniaturization, which occurs due to systemic androgens and genetic factors. The most common cause of hair loss in both genders is AGA. Prevalence differs according to ethnic groups. It is more common and more severe in white men than in Asian and black men. The incidence increases with age. According to Hamilton's study, the prevalence is $30 \%$ in men at the age of 30 , and $50 \%$ in the age of 50 . Generally, the age of onset is the $3^{\text {rd }}$ and $4^{\text {th }}$ decade, but hair loss can begin immediately after puberty and continues progressively. The phenotypes seen in men and women are different. In women, a diffuse dilution is observed while maintaining the frontal hairline. In men, the hairline is drawn backwards from the bitemporal regions and baldness appears in the vertex. Although clinical manifestations are different in both sexes, their pathogenesis is the same [1]. Although environmental factors on the severity of AGA cannot be revealed clearly, there are studies showing that it is more severe in overweight and simultaneous smokers [2]. At the same time, it was shown that both genders had more severe hair loss in 
patients with a family history and the disease started at an earlier age in male patients with a family history [3].

Although AGA is only a cosmetic problem, many studies have found the relationship between AGA and risk factors for cardiovascular disease or cardiovascular disease. In these patients, comorbidities such as hypertension, overweight, abnormal lipids, insulin resistance, carotid atheromatosis, and diabetes have been reported or increased risks of death from heart disease [4]. Metabolic syndrome is a combination of metabolic risk factors that pose a risk for type 2 diabetes and cardiovascular diseases. According to ATP 3 criteria, having at least three of the following 5 features is defined as metabolic syndrome [5]:

1. Abdominal obesity (waist circumference $\geq 102 \mathrm{~cm}$ in men, waist circumference $\geq 88 \mathrm{~cm}$ in women),

2. Serum triglyceride (TG) level $\geq 150 \mathrm{mg} / \mathrm{dL}$ or current drug use due to increased TG,

3. Serum high-density lipoprotein (HDL) cholesterol level $<40 \mathrm{mg} / \mathrm{dL}$ in men, $<50 \mathrm{mg} / \mathrm{dL}$ in women,

4. Blood pressure $\geq 130 / 85 \mathrm{mmHg}$ or current drug use for hypertension,

5. Fasting blood sugar $\geq 100 \mathrm{mg} / \mathrm{dL}$.

Various studies have investigated the possible relationship between AGA and metabolic syndrome. However, the relationship between AGA and the components of the metabolic syndrome has not been clearly established, as the results in the studies are contradictory. Many studies have been published examining the relationship between AGA and dyslipidemia, one of the criteria of metabolic syndrome. Some studies found a significant relationship, while others did not. Therefore, the relationship between AGA and dyslipidemia is still controversial. Our aim in this study is to reveal the relationship between AGA and dyslipidemia.

\section{Material and Methods}

This prospective study included male individuals between the ages of 18-30 who applied to the Istanbul University-Cerrahpasa, Cerrahpasa Faculty of Medicine Skin and Venereal Diseases outpatient clinic between December 1, 2017 and June 1, 2018. The study were approved by the Istanbul University-Cerrahpasa, Cerrahpasa Faculty of Medicine of Local Ethics Committee (protocol number: A-14, date: 02.01.2018).

Forty patients with stage 2 and higher AGA according to HamiltonNorwood scale in the clinical examination, as a patient group; 40 patients, without AGA on clinical examination or with the most stage 1 AGA according to the Hamilton-Norwood scale were accepted as the control group. Hamilton-Norwood stages of the patients were determined by clinical examination:
Type 1: There is no hairline retraction or minimal retraction in the frontotemporal region.

Type 2: The frontotemporal region hairline shows symmetrical and triangular retraction, although it is less common in the middle of the frontal region.

Type 3: This stage is the period when the presence of baldness becomes evident. There is symmetrical deep frontotemporal retraction.

Type 4: There is severe frontal and frontotemporal hair loss. Significant dilution is seen in the vertex. These two areas are separated along the hill with a thick headband.

Type 5: It is seen that the headband specified in type 4 becomes thinner. The hairless areas in the vertex and frontotemporal have increased.

Type 6: Regions expressed as hair bands have also been shed and the two regions merge.

Type 7: It is the most severe form. Only a narrow band of hair in a horseshoe shape remains on the sides and back of the scalp. This hair is usually not dense and may be quite fine.

Pull test was evaluated in patients with hair loss. For the pull test, approximately 50-100 hairs were held between the thumb and forefinger and gently pulled from the proximal to the distal. Easy removal of more than 6 hairs during this pull process has been interpreted as positive. In addition, body mass indexes (BMI), concomitant diseases of all patients, whether there is male-type shedding in the first degree relative are recorded in the files. Verbal and written consent was obtained from all participants by informing all patients about the purpose and method of the study. Fasting serum TG and HDL cholesterol values were studied in the blood taken from the patients participating in the study. The reference values are $150 \mathrm{mg} / \mathrm{dL}$ for TG and $40 \mathrm{mg} / \mathrm{dL}$ for HDL cholesterol. TG and HDL values were compared between these two groups and according to reference intervals.

Exclusion criteria from the study:

- Patient group outside the 18-30 age range,

- Patients with a different hair disease other than AGA,

- Those with systemic disease,

- Patients receiving medical treatment for dyslipidemia,

- Those with familiar lipid disease in the family,

- Patients who use drugs that can affect their blood fat,

- Patients with a BMI of 30 and above (obese).

\section{Statistical Analysis}

Descriptive statistical evaluations of all variables in the study were made by calculating the mean and standard deviation values. 
Mann-Whitney $U$ test was used to compare two independent groups, McNemar test was used to compare dependent groups, and Pearson chi-square analysis was used to analyze categorical data. All statistical analyzes were done with Statistical Package for the Social Science 21st edition (SPSS-21) program. In statistical decisions, $p<0.05$ level was accepted as an indicator of significant difference.

\section{Results}

The study included 40 patients in the patient group and 40 patients in the control group. The average age of the patient group is 23.12 and the average age of the control group is 21.95. There is no statistical difference between the two groups in terms of age distribution $(p<0.005)$.

According to the Hamilton-Norwood classification, 33 patients (82.5\%) were in stage 2, 5 patients (12.5\%) in stage 3, 1 patient $(2.5 \%)$ in stage 4, 1 patient (2.5\%) in stage 6. 'dr. While 28 patients (70\%) did not have AGA in the control group, the number of patients with stage 1 was 12 (30\%).

Fourteen people (35\%) in the patient group and 12 people (30\%) in the control group reported having AGA in their first degree relatives. There was no statistical difference between the two groups in terms of family history $(p<0.005)$.

While $24(60 \%)$ patients in the patient group did not have any additional dermatological diseases other than hair loss, acne vulgaris in 7 patients (17.5\%), seborrhea in 2 patients (5\%), seborrheic eczema in 5 patients (12.5\%), milium in 1 patient (12.5\%) and folliculitis in 1 patient (12.5\%). While 18 (45\%) patients in the control group did not have any additional dermatological diseases, 12 patients (30\%) had acne vulgaris, 6 patients (15\%) had seborrheic eczema, 2 patients (\%5) had tinea pedis, 1 patient (2.5\%) had folliculitis and 1 patient $(2.5 \%)$ had tinea versicolor. The most common comorbidity in both groups is acne vulgaris.

The average BMI of the patient group was 23.78. The average BMI of the control group is 23.60. There is no statistical difference between the two groups in terms of BMI $(p<0.005)$.

The mean of serum TG values in the patient group was 89.60. The mean of serum triglyceride values in the control group is 85.40 . There was no statistical difference between the two groups in terms of serum TG values $(p<0.005)$. The number of people with serum TG value $\geq 150 \mathrm{mg} / \mathrm{dL}$ is $5(12.5 \%)$ in the patient group and $3(7.5 \%)$ in the control group; however, this difference was not statistically significant $(p<0.005)$.

The average of serum HDL cholesterol values in the patient group was 52.67; the average of serum HDL cholesterol values in the control group is 52.62. There was no significant difference between serum HDL cholesterol values between both groups $(p<0.005)$. The number of people with serum HDL cholesterol value $<40 \mathrm{mg} / \mathrm{dL}$ is $4(10 \%)$ in the patient group and $1(2.5 \%)$ in the control group; however, this difference was not statistically significant $(p<0.005)$.

Since the number of patients with different AGA severity was not sufficient for each stage according to the Hamilton-Norwood classification in the patient group, the relationship between AGA severity and TG and HDL cholesterol values could not be evaluated.

In the patient group, the number of patients with positive pull test was 16 (40\%) and the number of patients with negative pull test was 24 (60\%). TG and HDL cholesterol values were compared between positive and negative patients with pull test, but no relation was found $(p<0.005)$ (Table 1).

\section{Discussion}

The pathophysiology of the relationship between AGA and dyslipidemia has not been clearly established. One of the most likely mechanisms to explain this relationship is altered peripheral sensitivity to androgens. Testosterone is converted to dihydrotestesterone with 5 alpha reductase enzymes in peripheral tissues and DHT has 5 times more affinity than androgen receptors. Five alpha reductase enzymes and androgen receptors are found in the arterial wall, blood vessels and adipose tissue. It has been suggested that increased sensitivity to androgens will be responsible for symptoms of AGA and metabolic syndrome. However, more research is needed on this subject. The second factor to explain the link between AGA and dyslipidemia is chronic microinflammation. Perifollicular inflammatory infiltration observed in the course of AGA may be a local reflection of systemic inflammation leading to the development of metabolic syndrome. Proinflammatory cytokines make serum lipid disorders by making changes in cholesterol transport and apolipoproteins [6].

In the metaanalysis published by Kim et al. [4] by analyzing 19 studies related to AGA and dyslipidemia, they stated that serum total cholesterol, TG and low-density lipoprotein (LDL) cholesterol levels were higher in AGA groups compared to control groups, and $\mathrm{HDL}$ cholesterol values were lower in AGA groups compared to control groups. These results indicate that AGA patients have a more atherogenic lipid profile and may partially explain the increased cardiovascular disease risk relationship in AGA patients. When subgroup analysis was performed by sex, higher total cholesterol, TG and LDL cholesterol levels were found in male patients and lower HDL levels were found in female patients compared to the control groups. However, the number of studies with women is very low and more studies are needed to evaluate the difference between genders.

The increased sensitivity of the scalp to androgens is part of the pathophysiology of AGA. Androgens affect the risk factors (hyperinsulinemia, glucose intolerance, high TG and low HDL 
Table 1. Demographic, clinical and laboratory data of patients

\begin{tabular}{|c|c|c|c|c|}
\hline & \multicolumn{2}{|c|}{ Patients (40) } & \multicolumn{2}{|c|}{ Control (40) } \\
\hline \multirow{4}{*}{$\begin{array}{l}\text { AGA severity according to Hamilton-Norwood } \\
\text { classification }\end{array}$} & Stage 2 & $33(82.5 \%)$ & Stage 1 & $12(30 \%)$ \\
\hline & Stage 3 & $5(12.5 \%)$ & No AGA & $28(70 \%)$ \\
\hline & Stage 4 & $1(2.5 \%)$ & & \\
\hline & Stage 6 & $1(2.5 \%)$ & & \\
\hline Age (years) (average) & \multicolumn{2}{|l|}{23.12} & 21.95 & \\
\hline BMI (kg/m²) (average) & \multicolumn{2}{|l|}{23.78} & 23.60 & \\
\hline Family story & \multicolumn{2}{|l|}{$14(35 \%)$} & $12(30 \%)$ & \\
\hline \multirow[t]{6}{*}{ Concomitant dermatological diseases } & $7(17.5 \%)$ & Acne vulgaris & $12(30 \%)$ & Acne vulgaris \\
\hline & $2(5 \%)$ & Seborrhea & $6(15 \%)$ & Seborrheic eczema \\
\hline & $5(12.5 \%)$ & Seborrheic eczema & $2(5 \%)$ & Tinea pedis \\
\hline & $1(2.5 \%)$ & Milium & $1(2.5 \%)$ & Folliculitis \\
\hline & $1(2.5 \%)$ & Folliculitis & $1(2.5 \%)$ & Tinea versicolor \\
\hline & $24(60 \%)$ & No additional diseases & $18(45 \%)$ & No additional diseases \\
\hline TG value mg/dL (average) & \multicolumn{2}{|l|}{89.60} & 85.40 & \\
\hline Number of patients with TG value $\geq 150 \mathrm{mg} / \mathrm{dL}$ & \multicolumn{2}{|l|}{$5(12.5 \%)$} & $3(7.5 \%)$ & \\
\hline HDL cholesterol value mg/dL (mean) & \multicolumn{2}{|l|}{52.67} & 52.62 & \\
\hline Number of patients with $\mathrm{HDL}$ value $<40 \mathrm{mg} / \mathrm{dL}$ & \multicolumn{2}{|l|}{$4(10 \%)$} & $1(2.5 \%)$ & \\
\hline
\end{tabular}

cholesterol values, hypertension and obesity) determined for coronary heart disease. The epidemiological relationship between AGA and coronary heart diseases is assumed to be based on these risks. While some studies support this relationship, some studies do not. Giltay et al. [7] followed 81 transsexual patients who switched from female to male, using testosterone esters in this process. In $38.3 \%$, AGA developed, but they did not notice a difference in their blood fat or weight. In our study, to support this study, no difference was found between the patient and control groups between TG and HDL cholesterol values and BMI.

Epidemiological studies show that male patients with AGA have higher insulin resistance and coronary heart disease. Insulin resistance has an additional pathogenetic role in hair follicle maturation. Vasoactive substances released due to endothelial dysfunction in insulin resistance cause perifollicular vasoconstriction and proliferation in smooth muscle cells in the vascular wall. As a result, microvascular circulation is impaired, local tissue hypoxia occurs, and a progressive miniaturization is observed in the hair follicles.

Mumcuoglu et al. [8] conducted a study evaluating the insulin resistance and presence of metabolic syndrome in male patients with early-onset AGA (at least stage 3 according to the HamiltonNorwood scale) compared to the control group. According to the results of this study, no difference was observed in fasting glucose, insulin, TG, HDL cholesterol and oral glucose tolerance tests between the two groups. However, HOMA and FIRI indices showing insulin resistance in AGA group are higher than control group. In addition, diastolic blood pressure and total cholesterol levels were higher in the AGA group. Considering the metabolic syndrome criteria, there is no difference between the two groups. In our study, we compared TG and HDL levels, one of the criteria of metabolic syndrome, between patients with and without AGA. As in this study, we did not include obese patients (patients with a BMI above 30) in our study to eliminate the weight factor, which can affect blood fat. In addition, the age ranges of the patient and control groups are the same with this study. In our study, the difference between the blood fats between the two groups supports this study. However, in our study, 33 patients (82.5\%) were stage 2, 5 patients (12.5\%) were stage 3, 1 patient (2.5\%) was stage 4, 1 patient (2.5\%) was stage 6 . In other words, the number of patients included in the early-onset AGA classification is only 7 (17.5\%). Since the number of patients with different AGA severity in the patient group was not sufficient for each stage, we could not evaluate the relationship between AGA severity and TG and HDL cholesterol values.

In a study by Gopinath and Upadya [9], 85 patients diagnosed with early-onset AGA between the ages of 18-55 and 85 male patients without hair loss were compared in terms of metabolic syndrome criteria. Metabolic syndrome was $22.4 \%$ in the AGA group and $9.4 \%$ in the control group. The frequency of abdominal obesity, hypertension is significantly higher and HDL cholesterol values are lower in patients with AGA compared to the control group. Based 
on this study data, researchers stated that screening would be beneficial especially in patients with early-onset AGA in terms of metabolic syndrome.

In another study examining the relationship between metabolic syndrome and AGA, 740 men aged 40-91 years in a community were evaluated for the presence of AGA and metabolic syndrome criteria. There was a significant relationship between the presence of AGA and metabolic syndrome. In addition, those who meet the metabolic syndrome criteria more frequently have a higher risk of AGA. Among the metabolic syndrome criteria, the most important factor associated with AGA was low HDL cholesterol. No relationship was found between other lipid levels and AGA. Considering the relationship between AGA severity and metabolic syndrome, patients with severe AGA ( $\mathrm{N}-\mathrm{H}$ stage 5 and above) are 2.6 times more likely to experience metabolic syndrome than moderate AGA patients ( $\mathrm{N}-\mathrm{H}$ stage $3-4)$. People with metabolic syndrome have a higher risk of having stage 4 and above AGA than those without metabolic syndrome. An inverse relationship was detected between HDL values and AGA severity. In the light of all these results, it has been said that individuals with moderate and severe AGA (N-H stage 3 and above) have a higher risk of dyslipidemia and metabolic syndrome and screening can be done in this direction [10].

In a study by Chakrabarty et al. [11], it was stated that metabolic syndrome is seen more in those with AGA. However, in this study, no relation was found between AGA severity and metabolic syndrome. This result may indicate that there is no relation, or it may be due to insufficient sample size to define this relation precisely. In the study of Chakrabarty et al. [11], it was reported that those with AGA also had higher TG values, higher blood pressure and lower HDL cholesterol levels. The results in our study do not support this study. However, the number of patients in the patient and control group in our study is very low compared to this study. At the same time, our study was conducted only among individuals applying to the hospital. Therefore, it is possible that our sample set does not represent the whole society.

Although the pathogenetic mechanism of atherosclerosis is well known, the relationship between alopecia and atherosclerosis is not clear. High cholesterol and TG levels are involved in the process, along with other mechanisms that initiate atheromatosis. On the other hand, HDL cholesterol protects the vascular wall from aggressive factors (endothelial adhesion, migration of monocytes, etc.) and provides reverse transport of cholesterol. The relationship between lipid disorders and coronary heart disease in patients with male and female AGA is explained through these mechanisms [12]. In one study, blood lipid levels of 150 patients (80 men and 70 women) aged 35-60 with early onset AGA and 150 control patients (80 men and 70 women) who presented for other skin diseases were compared. In patients with AGA, significantly higher TG values, total cholesterol values, LDL values and lower HDL values were found compared to the control group. In men with AGA, significantly higher TG values, total cholesterol values and LDL values were observed compared to the control group. However, there was no significant difference in HDL values in male patients compared to the control group. The frequency of dyslipidemia was found to be higher in women and men with AGA. No significant relationship was observed between lipid parameters and AGA severity in male and female patients [12].

In a study by Sadighha and Zahed [13], in patients with and without AGA, a significantly higher TG and total cholesterol/HDL-cholesterol ratio was found in men with AGA and HDL values were found to be significantly lower.

According to the Hamilton-Norwood scale, patients with stage 3 vertex or more severe hair loss before age 35 are classified as early-onset AGA. Some authors have suggested that early-onset AGA is genetically different from late-onset. Matilainen et al. [14] conducted a community-based case-control study to assess whether early-onset AGA is a risk factor for early-onset and severe coronary artery disease requiring surgical intervention. In this study, 85 male patients who had undergone coronary surgery in a region with a population of 7200 were included and an agematched control group was established for each case. Patients were compared in terms of BMI, blood pressures, serum total cholesterol, TG, HDL-cholesterol, glucose levels. It was observed that the rates of dyslipidemia in patients with AGA were higher than the control groups. Patients with early and late-onset AGA were evaluated for having early or late coronary revascularization surgery. Male patients with early-onset AGA were found to have a higher rate of undergoing coronary surgery under the age of 60 than those with late-onset AGA or normal hair. This study supports the hypothesis that early-onset AGA may be an indicator of impaired endothelial function, the main pathogenetic mechanism of atherosclerosis.

Sharma et al. [15] examined the relationships between AGA and coronary heart disease risks, with 100 male patients aged 25-40 years old with Hamilton-Norwood stage 2 and above were compared with the control group. In the group with AGA, significantly higher TG, total cholesterol, LDL cholesterol, VLDL cholesterol and lower HDL cholesterol values were found compared to the control group, and these values were observed to increase with increasing AGA severity (HDL decreased with increasing AGA severity). Similar to this study, we compared male patients with Hamilton-Norwood stage 2 and above with the control group. However, in our study, the age range included younger patients than this study. In our study, we found TG and HDL cholesterol 
levels similar between patient and control groups. This may be because patients and controls are at a younger age compared to this study. In our study, we could not evaluate the relationship between AGA severity and TG and HDL cholesterol values since the number of patients with different AGA severity was not sufficient for each stage.

As well as the pathophysiological mechanisms responsible for the relationship between AGA and dyslipidemia, the effect of this relationship on treatment is a matter of curiosity. Statins are drugs that reduce the level of serum cholesterol that acts by inhibiting the 3-hydroxy-3-methylglutaryl coenzyme A (HMG-CoA) enzyme. Statins also have immune regulatory effects by inhibiting major histocompatibility complex class 2 mediated antigen presentation, preventing mast cell activation, stimulating regulatory $\mathrm{T}$ cells, and regulating the Th1/Th2 balance. In addition, statins have pleiotropic effects such as improving endothelial function, reducing oxidative stress, inflammation and thrombogenic response [16].

Finasteride, used in the treatment of AGA at a dose of $1 \mathrm{mg} /$ day, is a 5 alpha reductase (type 2) enzyme inhibitor. In a study that examined whether finasteride therapy in AGA reduced dihydrotestesterone levels and improved some metabolic syndrome criteria that may be associated with this condition, 12 early-onset AGA patients who received finasteride therapy for 12 months were followed. As expected in this process, serum dihydrotestesteron levels decreased, serum testosterone, androstenedione levels increased and free testosterone index increased. An increase in serum total cholesterol, HDL and LDL cholesterol was observed initially and these levels remained constant during treatment. There was a significant decrease in $\mathrm{HbA1c}$ levels, and a decrease in the limit of insulin resistance, which was evaluated using the constant for plasma glucose disappearance-KITT, was detected [17].

\section{Conclusion}

There are many studies in the literature examining the relationship between AGA and dyslipidemia, one of the criteria of metabolic syndrome. While most studies have found a significant relationship, data in some studies do not support this. Therefore, the relationship between AGA and dyslipidemia is still controversial. It is suggested that due to this relationship between AGA and metabolic syndrome, patients have a higher risk of coronary heart disease and type 2 diabetes, and screening for these patients will be beneficial. In our study, unlike most studies in the literature, no relationship was found between the presence of AGA and dyslipidemia. However, more studies with randomized controlled, larger patient series are needed to resolve the debate on this issue.

\section{Ethics}

Ethics Committee Approval: The study were approved by the Istanbul University-Cerrahpasa, Cerrahpasa Faculty of Medicine of Local Ethics Committee (protocol number: A-14, date: 02.01.2018).

Informed Consent: Consent form was filled out by all participants.

Peer-review: Internally peer-reviewed.

\section{Authorship Contributions}

Surgical and Medical Practices: T.A., Z.K., Ö.A., Concept: T.A., Z.K., Ö.A., Design: T.A., Z.K., Ö.A., Data Collection or Procesfsing: T.A., Z.K., Ö.A., Analysis or Interpretation: T.A., Z.K., Ö.A., Literature Search: T.A., Z.K., Ö.A., Writing: T.A., Z.K., Ö.A.

Conflict of Interest: No conflict of interest was declared by the authors.

Financial Disclosure: The authors declared that this study received no financial support.

\section{References}

1. Salman KE, Altunay IK, Kucukunal NA, Cerman AA. Frequency, severity and related factors of androgenetic alopecia in dermatology outpatient clinic: Hospital-based cross-sectional study in Turkey. An Bras Dermatol 2017;92:35-40.

2. Fortes, C, Mastroeni S, Mannooranparampil TJ, Ribuffo M. The combination of overweight and smoking increases the severity of androgenetic alopecia. Int J Dermatol 2017;56:862-867.

3. Yeo IK, Jang WS, Min PK, Cho HR, Cho SW, Hong NS, Kang JS, Ki DH, Kim HJ, Kim YC, Kim YS, Lee IJ, Lee SW, Lim ES, Moon DC, Nam KH, Oho CK, Park SW, Shin KS, Yoo HC, Hong CK. An epidemiological study of androgenic alopecia in 3114 Korean patients. Clin Exp Dermatol 2014;39:25-29.

4. Kim MW, Shin IS, Yoon HS, Cho S, Park HS. Lipid profile in patients with androgenetic alopecia: a meta-analysis. J Eur Acad Dermatol Venereol 2017;31:942-951.

5. Expert Panel on Detection, Evaluation, and Treatment of High Blood Cholesterol in Adults. Executive Summary of The Third Report of The National Cholesterol Education Program (NCEP) Expert Panel on Detection, Evaluation, And Treatment of High Blood Cholesterol In Adults (Adult Treatment Panel III). JAMA 2001;285:2486-2497.

6. Rudnicka L, Rakowska A. Dyslipidemia in patients with androgenetic alopecia. Statins, finasteride or both? J Eur Acad Dermatol Venereol 2017;31:921-922.

7. Giltay EJ, Toorians AWFT, Sarabdjitsingh AR, Vries NA De, Gooren LJG. Established risk factors for coronary heart disease are unrelated to androgeninduced baldness in female-to-male transsexuals. J Endocrinol 2004;180:107112.

8. Mumcuoglu C, Ekmekci TR, Uca S. The investigation of insulin resistance and metabolic syndrome in male patients with early-onset androgenetic alopecia. Eur J Dermatol 2011;21:79-82.

9. Gopinath H, Upadya GM. Metabolik Syndrome in androgenic alopecia. Indian J Dermatol Venereol Leprol 2016;82:404-408.

10. Su LH, Chen TH. Association of androgenetic alopecia with metabolic syndrome in men: A community-based survey. Br J Dermatol 2010;163:371377.

11. Chakrabarty S, Hariharan R, Gowda D, Suresh H. Association of premature androgenetic alopecia and metabolic syndrome in a young Indian population. Int J Trichology 2014;6:50-53. 
12. Arias-Santiago S, Gutiérrez-Salmerón MT, Buendía-Eisman A, Girón-Prieto MS, Naranjo-Sintes R. A comparative study of dyslipidaemia in men and women with androgenic alopecia. Acta Derm Venereol 2010;90:485-487.

13. Sadighha A, Zahed GM. Evaluation of lipid levels in androgenetic alopecia in comparison with control group. J Eur Acad Dermatol Venereol 2009;23:8081.

14. Matilainen VA, Mäkinen PK, Keinänen-Kiukaanniemi SM. Early onset of androgenetic alopecia associated with early severe coronary heart disease: A population-based, case-control study. J Cardiovasc Risk 2001;8:147-151.
15. Sharma L, Dubey A, Gupta PR, Agrawal A. Androgenetic alopecia and risk of coronary artery disease. Indian Dermatol Online J 2013;4:283-287.

16. Liao JK, Laufs U. Pleiotropic effects of statins. Annu Rev Pharmacol Toxicol 2005;45:89-118.

17. Duskova M, Hill M, Starka L. Changes of metabolic profile in men treated for androgenetic alopecia with $1 \mathrm{mg}$ finasteride. Endocr Regul 2010;44:3-8. 